Revue d'histoire de l'Amérique française

7S REVUE D.HISTOIRE DE L'AMÉRIQUE FRANÇAISE

\title{
Aux origines d'une population régionale : mythes et réalités démographiques et sociales
}

\section{Gérard Bouchard et Lise Bergeron}

Volume 42, numéro 3, hiver 1989

URI : https://id.erudit.org/iderudit/304707ar

DOI : https://doi.org/10.7202/304707ar

Aller au sommaire du numéro

\section{Éditeur(s)}

Institut d'histoire de l'Amérique française

\section{ISSN}

0035-2357 (imprimé)

1492-1383 (numérique)

Découvrir la revue

\section{Citer cet article}

Bouchard, G. \& Bergeron, L. (1989). Aux origines d'une population régionale : mythes et réalités démographiques et sociales. Revue d'histoire de l'Amérique française, 42(3), 389-409. https://doi.org/10.7202/304707ar
Résumé de l'article

Prenant prétexte de la célébration du $150^{\mathrm{e}}$ anniversaire de la région du Saguenay, cet article veut contribuer à mettre en lumière la confusion, les inexactitudes et les mythes que peuvent susciter la naissance et l'évolution d'une population régionale. L'historiographie populaire qui s'en nourrit et qui les perpétue semble étrangement imperméable aux enseignements de l'enquête scientifique, pratiquée selon les règles du métier d'historien.

Les auteurs s'appliquent ici à clarifier certains points relatifs à la formation de la population saguenayenne : d'abord en mettant de l'avant quelques concepts permettant de mieux cerner les questions à l'étude, puis, à l'aide de données tirées du fichier-réseau de SOREP, en rectifiant quelques stéréotypes reliés à la dynamique de cette population régionale.

Les principaux résultats portent sur la mise en place du premier noyau d'immigrants, leur enracinement, leur descendance immédiate et leur contribution - par ailleurs très inégale - à l'accroissement et au renouvellement de la population sur six générations.
Tous droits réservés @ Institut d'histoire de l'Amérique française, 1989

Ce document est protégé par la loi sur le droit d'auteur. L'utilisation des services d'Érudit (y compris la reproduction) est assujettie à sa politique d'utilisation que vous pouvez consulter en ligne.

https://apropos.erudit.org/fr/usagers/politique-dutilisation/ 


\title{
AUX ORIGINES D'UNE POPULATION RÉGIONALE: MYTHES ET RÉALITÉS DEMOGRAPHIQUES ET SOCIALES ${ }^{1}$
}

\author{
GÉRARD BOUCHARD \\ LISE BERGERON \\ Centre interuniversitaire SOREP \\ Université du Québec à Chicoutimi
}

\section{RÉSUMÉ}

Prenant prétexte de la célébration du 150e anniversaire de la région du Saguenay, cet article veut contribuer à mettre en lumière la confusion, les inexactitudes et les mythes que peuvent susciter la naissance et l'évolution d'une population régionale. L'historiographie populaire qui s'en nourrit et qui les perpétue semble étrangement imperméable aux enseignements de l'enquête scientifique, pratiquée selon les règles du métier d'historien.

Les auteurs s'appliquent ici à clarifier certains points relatifs à la formation de la population saguenayenne: d'abord en mettant de l'avant quelques concepts permettant de mieux cerner les questions à l'étude, puis, à l'aide de données tirées du fichier-réseau de SOREP, en rectifiant quelques stéréotypes reliés à la dynamique de cette population régionale.

Les principaux résultats portent sur la mise en place du premier noyau d'immigrants, leur enracinement, leur descendance immédiate et leur contribution — par ailleurs très inégale — à l'accroissement et au renouvellement de la population sur six générations.

\section{ABSTRACT}

In the wake of the 150th anniversary of the Saguenay region and the celebrations that surrounded the event, this paper aims to highlight the confusion, inaccuracies and myths that can infiltrate the popular accounts of the beginnings and evolution of a regional population. These myths and clichés feed a popular historiography that perpetuate them and seems quite impervious to the evidence produced by scientific research, as practiced by modern professional historians.

Here, the authors intend to clarify some points pertaining to the formation of the Saguenay population; by a) setting forth a few concepts appropriate to better identify the issues to be investigated; $b$ ) producing new evidence drawn from the SOREP database. Thus, the authors hope to get rid of some old stereotypes said to account for the dynamics of this regional population.

The main findings pertain to the establishment of the first settlers, their ability to take roots, their fertility and their unequal contribution to the population growth over six generations.

1 Les travaux qui ont conduit à cet article ont été réalisés grâce à l'appui financier du Fonds FCAR (Québec), du Conseil de recherches en sciences humaines du Canada (Ottawa) et de l'Université du Québec à Chicoutimi. Les auteurs remercient Jeannette Larouche, Bernard Casgrain, Michel Hubert et Alain Simard qui ont contribué à la production et à l'élaboration des données. 


\section{1 - DES MÉPRISES DE LA MÉMOIRE COLLECTIVE}

La région du Saguenay fêtait en 1988 le 150e anniversaire de sa fondation - entendons par là: les débuts des défrichements et de la colonisation par les Blancs. Les célébrations et les rappels auxquels cette circonstance a donné lieu fournissent un double objet de réflexion à l'historien. C'est d'abord, sur un plan strictement historique, l'événement lui-même de la naissance d'une région. Encore trop près de nos racines, il n'est pas certain que nous percevions tout l'intérêt de ce genre de création collective largement pénétrée par la mythologie des recommencements. C'est, en deuxième lieu, et sur un plan historiographique cette fois, l'ensemble des représentations qui sont proposées pour en rendre compte et qui sont accréditées par une tradition locale écrite aussi bien qu'orale.

Ainsi, la plupart des Saguenayens ont appris à un moment ou l'autre que les fameux Vingt-et-Un, originaires de Charlevoix, étaient les valeureux fondateurs du Saguenay - de même que, selon le goût du jour, l'industriel William Price, l'ex-agent de la Hudson Bay Peter McLeod, et Mgr Racine; à moins que ce ne soient les marchands de fourrures du XVIIe siècle, selon une thèse qui n'a peut-être pas reçu toute l'attention qu'elle méritait ${ }^{2}$. C'est à la Baie des Hahas, aussi, que l'on trouve le monument dressé à la mémoire des fondateurs, là même où ils auraient débarqué. La plupart savent par ailleurs que l'immense majorité des habitants d'aujourd'hui descend de cette poignée d'immigrants, ce qui explique l'homogénéité de la population, formée comme on sait, d'une grande partie de Tremblay, peut-être même à plus de la moitié?... Il est également bien connu que l'éloignement dont la région a souffert a compromis l'immigration et a poussé aux mariages consanguins, lesquels y étaient beaucoup plus fréquents qu'ailleurs au Québec. On tiendrait là du reste la cause de l'incidence très élevée des maladies génétiques dans cette partie de la province. Enfin, et même si de nombreuses familles en garderaient encore le secret, il est de notoriété qu'au gré de circonstances où la moralité perd un peu son compte, le sang amérindien se serait infiltré dans la majorité des familles; et ce serait là l'origine de certains traits du caractère saguenayen...

Pour une part, ces représentations sont soit carrément inexactes ou simplement non fondées, dans l'état présent de la recherche. Mais chacune a son histoire et serait susceptible d'une analyse qui, dans chaque cas, éclairerait un lieu - grand ou petit - de la culture régionale. Parmi les inexactitudes, il faut de toute évidence ranger le modèle de la reproduction en vase clos (sans apports migratoires), l'homogénéité exceptionnelle du bassin patronymique, la fréquence des mariages

2 Lorenzo Angers, Chicoutimi poste de traite, 1676-1856 (Ottawa, Éditions Leméac, coll. «Historique», 1971), $123 \mathrm{p}$. 
LA RÉGION DU SAGUENAY ET SES SOUS-RÉGIONS

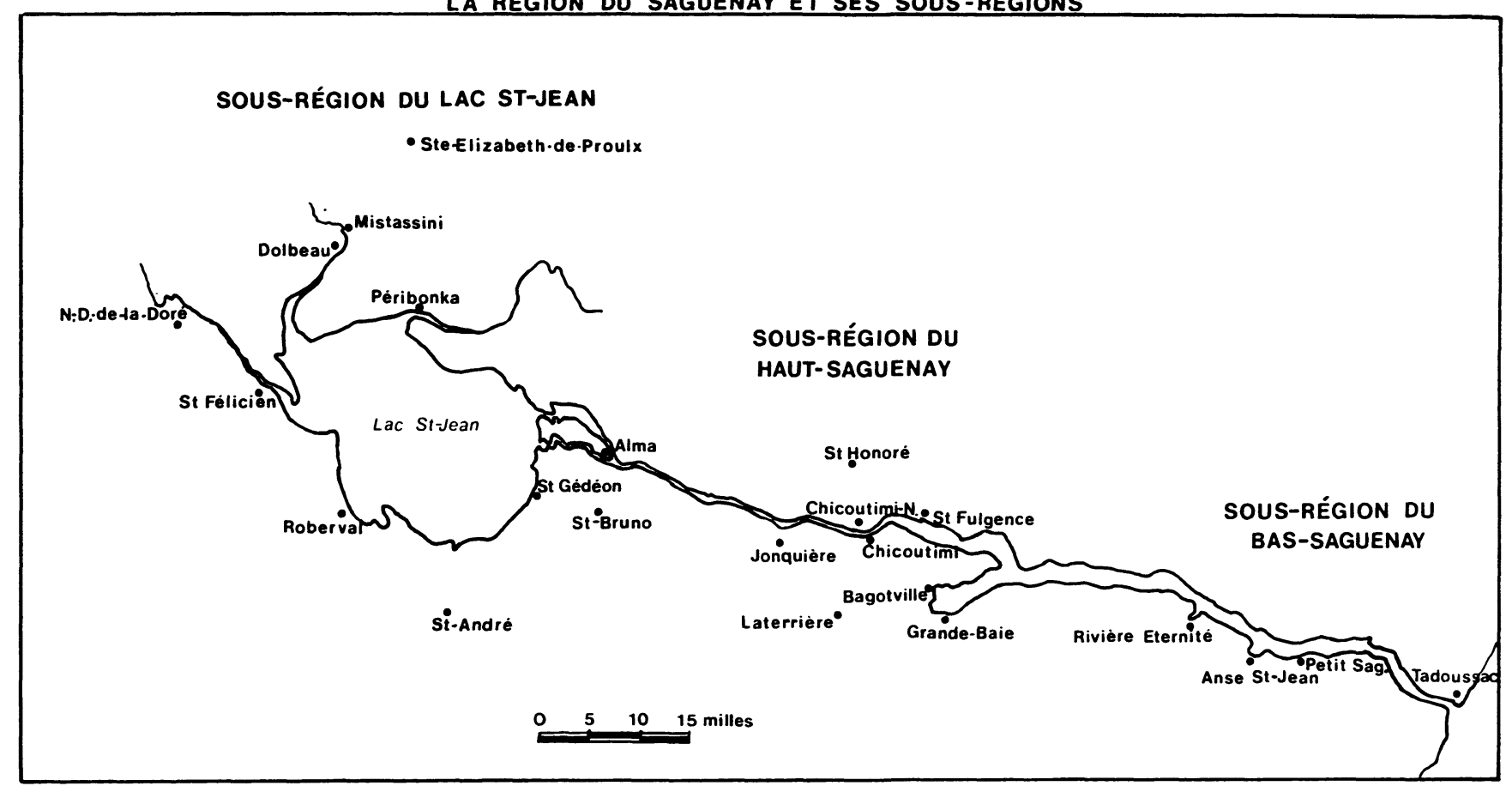


consanguins, le caractère ponctuel de l'immigration initiale ${ }^{3}$. Dans la même veine, bien que sur un plan plus anecdotique, il faut bien s'étonner un peu aussi de ce que la Baie des Hahas ait toujours été reconnue et célébrée comme le berceau du Saguenay alors que, comme l'ont pourtant écrit les historiens locaux eux-mêmes depuis quelques décennies, les premiers établissements ont eu lieu à l'Anse Saint-Jean, à quelque 50 kilomètres à l'est (carte). Parmi les représentations non fondées, compte tenu de l'état actuel des connaissances, on retrouve en particulier la fascination ambiguë que continuent d'exercer les Autochtones sur les Blancs. Ainsi, il n'existe actuellement aucune donnée empirique rigoureuse qui pourrait accréditer l'idée des nombreuses unions entre Blancs et Amérindiens au Saguenay au XIXe siècle ${ }^{4}$. C'est du reste une question à laquelle il sera difficile de répondre, les sources documentaires appropriées faisant défaut. Deux recherches sont cependant en cours au sein de SOREP sur ce sujet, l'une à partir du fichier de population, l'autre sur le terrain de la génétique moléculaire (analyse comparée des systèmes HLA).

Dans le cadre de cet essai, en plus de remettre en cause certains points de l'historiographie régionale, nous voulons mieux cerner les effectifs démographiques qui sont à l'origine du Saguenay contemporain, notre période de référence débutant avec la colonisation et les premiers défrichements effectués par des immigrants de Charlevoix en 1838. Nous essaierons aussi de mesurer la contribution de ces immigrants primitifs à la formation et à la reproduction de la population régionale.

Il est utile de fixer d'abord quelques concepts relatifs aux débuts du peuplement. Pour une part en effet, les critiques que nous adressons à l'historiographie tiennent à la confusion ayant existé entre cinq réa-

3 Nous en avons fait justice, ailleurs. Cf. Gérard Bouchard, Claude Laberge, Charles R. Scriver, Francis Glorieux et al., «Étude démographique et généalogique de deux maladies héréditaires au Saguenay», Cahiers québécois de démographie, 13,1 (avril 1984): 118-137; Gérard Bouchard et Raymond Roy, «Genetic Studies in the Province of Quebec, Using a Computerized Population Register», Proceedings of the Workshop on Computerized Record Linkage in Health Research/Débats de l'Atelier sur le jumelage informatisé des données nominatives pour fin de recherche dans le domaine de la santé, Ottawa, Ontario (21-23 mai 1986): 131-148; Gérard Bouchard, Claude Laberge et Charles R. Scriver, «Reproduction démographique et transmission génétique dans le nord-est de la province de Québec (18e-20e s.)», European Journal of Population, 4 (1988): 39-67. Aussi, Danielle Gauvreau, René Jetté et Mario Bourque, «Migration in the Saguenay Region: Evidence from Reconstituted Families, 1838-1911» Historical Methods, 20,4 (Fall 1987): 143-154.

On a toutefois étudié les fréquences géniques reliées aux groupes sanguins chez les Montagnais de Schefferville (Côte-Nord/Nouveau-Québec). Cet indicateur a conduit à la conclusion que les Montagnais étaient sans doute très différents de la population blanche du Saguenay. Voir B. S. Blumberg et al., «Blood Groups of the Naskapi and Montagnais Indians of Schefferville, Québec», Human Biology, 36 (1964): 246-272; Claude Magnan et Jean Benoist, «Les groupes sanguins des Canadiens français: la région du Saguenay-Lac Saint-Jean», F. Auger, J. Benoist et al., dir., Anthropologie biologique au Québec (Montréal, département d'anthropologie, Université de Montréal, 1977), 33-60. 
lités ou questions différentes. Il y a lieu de distinguer en premier lieu les promoteurs. Par intérêt économique, social ou autre, ils ont contribué, principalement de l'extérieur, à l'ouverture de la région par des démarches de propagande, de levée de fonds, de pression sur les gouvernants, de création de sociétés, etc. Il y a en deuxième lieu les immigrants, ceux qui sont venus s'établir dans la région pour une période allant de quelques mois à plusieurs décennies. En troisième lieu, on distingue les pionniers proprement dits. Ce sont ceux qui sont venus prendre résidence et qui ont contribué sur place au développement agricole, industriel, culturel ou autre - de la nouvelle société. Parmi ces pionniers, un certain nombre sont des ancêtres de la population saguenayenne, du fait qu'ils ont simplement laissé des descendants. Enfin, à un cinquième plan, il faut accorder une place importante à la notion de fondateurs, empruntée à la génétique, pour désigner les personnes qui ont apporté une contribution importante au bassin génique régional. En effet, à cause des hasards de la dérive, un ancêtre peut compter plusieurs descendants dans une population après cinq ou six générations, sans avoir apporté à leur génotype une contribution équivalente; il convient donc de distinguer les ancêtres et les fondateurs.

$\mathrm{Au}$ total, ce sont bien là cinq réalités spécifiques, toutes inhérentes au fait de la naissance ou de la création d'une société neuve: promotion ou organisation, déplacement de population et résidence, implantation et développement, reproduction démographique et transmission génétique. Par ailleurs, il est évident que ces rôles peuvent être cumulés, tout comme on peut être, par exemple, promoteur ou immigrant sans être pionnier.

\section{2 - REVUE DE L'HISTORIOGRAPHIE SAGUENAYENNE}

Rappelons que le Saguenay a accueilli ses premiers défricheurs en 1838. Son territoire avait été sillonné depuis le XVIIe siècle par des explorateurs, des missionnaires, des commerçants de fourrures. Mais ces activités n'avaient pas donné lieu à une véritable occupation agraire. Jusqu'en 1842 officiellement, il a été tenu à bail par diverses compagnies - la dernière étant la Hudson Bay - et réservé au commerce des fourrures, une clause interdisant les défrichements et l'établissement de colons. Néanmoins, dès le printemps 1838, des immigrants de Charlevoix occupent et défrichent illégalement des terres à l'Anse-SaintJean puis à la Baie des Hahas ${ }^{5}$. Ce courant migratoire n'a pu être endigué, malgré l'opposition initiale de la Compagnie: entre 1838 et 1871, 3761 personnes immigrèrent au Saguenay, parmi lesquelles $73 \%$ provenaient

5 Sur ce sujet, voir Gérard Bouchard, «Le peuplement blanc», C. Pouyez et al., Les Saguenayens. Introduction à l' histoire des populations du Saguenay, XVIe-XXe siècles (Québec, Presses de l’Université du Québec, 1983), 396 p. Voir chapitre 4. 
de Charlevoix ${ }^{6}$. Faisant montre par ailleurs d'une fécondité très élevée, la population de la nouvelle région se chiffrait à 17493 en 1871 et à 37367 à la fin du siècle 7 . Parallèlement, le peuplement progressait d'est en ouest, atteignant durant la décennie 1851-1861 le lac SaintJean, qu'il contourna grosso modo dans le sens des aiguilles d'une montre.

Pour ce qui concerne les premiers établissements, l'intérêt qu'il y a à identifier et à compter avec précision pionniers, immigrants ou fondateurs relève moins de la curiosité anecdotique ou commémorative que d'une nécessité de l'enquête sociale, démographique et génétique. En effet, pour mieux comprendre le type de communauté qui s'élabore, il est utile de connaître l'origine, le profil ethnique, le statut familial et la condition socio-économique des premiers immigrants ${ }^{8}$. Afin de reconstituer le modèle de développement de la population ou pour inférer la composition du pool génique, il est encore nécessaire de bien connaître ses premiers contributeurs. C'est pourquoi nous avons cherché à éclairer le premier transfert de population entre Charlevoix et Saguenay à partir de 1838 .

Les historiens du Saguenay qui se sont penchés sur cette période insistent sur le rôle central joué par les membres de la Société des Vingtet-Un, fondée en 1837 pour effectuer, avec l'accord de la Compagnie de la Baie d'Hudson, des opérations de coupe forestière au Saguenay. Cette société comprenait 21 actionnaires et 16 associés ou co-actionnaires, lesquels - les premiers surtout - constituent un symbole très populaire de la fondation du Saguenay, sur lequel les écoliers sont régulièrement invités à disserter' ${ }^{9}$. Il n'est pas inutile de noter toutefois que, d'un auteur à l'autre, il y a certaines discordances dans la liste de ces présumés pionniers ${ }^{10}$.

\footnotetext{
6 Danielle Gauvreau, Le peuplement du Saguenay au 19e siècle: mesure et caractéristiques du mouvement d'immigration jusqu'en 1911. Communication présentée au congrès de la Société historique du Canada à Hamilton (juin 1987).

7 Christian Pouyez, Yolande Lavoie, Gérard Bouchard, Raymond Roy et al., Les Saguenayens. Introduction à l'histoire des populations du Saguenay, XVI-XXe siècles (Québec, Presses de l'Université du Québec, 1983), 386 p.

8 Par exemple, Gérard Bouchard et Jeannette Larouche, «Dynamique des populations locales: la formation des paroisses rurales au Saguenay (1840-1911)», Revue d'histoire de l'Amérique française, 41,3 (hiver 1988): 363-388.

Il existe même encore aujourd'hui un organisme honorifique appelé l'Ordre des Vingt-etUn, dont le but est de consacrer les mérites des Saguenayens d'exception.

10 Voir la liste donnée par Victor Tremblay, Histoire du Saguenay depuis les origines jusqu'à 1870 (Chicoutimi, La Librairie Régionale, Inc., 1968), 465 p., qui, étrangement, n'en énumère que 19. En outre, cette liste ne contient pas les mêmes noms que celle publiée par Léonidas Bélanger, La Société des Vingt-et-Un (conférence prononcée à Chicoutimi, le 25 février 1983). Assez curieusement aussi, ce dernier a publié une autre version de la liste qui, au chapitre des co-associés, diffère de la précédente: Léonidas Bélanger, «La Société des Vingt-et-Un», Saguenayensia, 25,2 (1983): 45-49. Pour les fins de cette recherche, nous nous en sommes remis à Mario Lalancette, «Alexis Tremblay Picoté (1787-1859)», Charlevoix, 2,1 (1987): 10.
} 
On apprend ainsi qu'en avril 1838, une goélette quittait La Malbaie ayant à son bord les premiers immigrants de Charlevoix, qu'une partie des passagers furent débarqués à l'Anse-Saint-Jean - vraisemblablement dans le courant du mois de mai - et qu'un groupe d'hommes $\mathrm{s}^{\prime} y$ trouvaient déjà, venus également de Charlevoix par la terre ferme ${ }^{11}$. On ne connaît donc ni l'importance ni les membres de ces premiers contingents. Début juin de la même année, un détachement de 14 hommes, dont on ignore aussi l'identité, aurait quitté l'Anse-Saint-Jean pour prendre pied à la Baie des Hahas, à l'endroit actuel de SaintAlexis-de-Grande-Baie ${ }^{12}$. Par ailleurs en octobre 1838, 48 personnes, parmi lesquelles douze couples ont pu être identifiés, arrivent pour s'établir à la Baie $^{13}$. S'agissant enfin de la croissance de la population, d'après quelques rapports de mission aujourd'hui introuvables ${ }^{14}$ et cités par divers auteurs qui, encore une fois, ne donnent pas de précision sur leur source, on aurait dénombré 336 personnes le long du Saguenay à la fin de 1839 et un millier à la Baie des Hahas en 1843.

À tout prendre, il reste beaucoup de travail à faire sur les modalités de la formation de la population régionale. Travail de découverte d'abord, mais aussi de remise en ordre. Par exemple, il faut sans doute rejeter l'idée, accréditée par R. Blanchard ${ }^{15}$, selon laquelle la colonisation du Saguenay aurait été avant tout coopérative, «lancée par des sociétés et non par des individus». C'est donner trop d'importance à la Société des Vingt-et-Un - qui a été du reste dissoute dès 1843 - et aux autres organismes de ce genre qui naîtront plus tard. L'ampleur du mouvement d'immigration originant de toutes les parties de Charlevoix, les motifs qui ont poussé les familles à quitter, les conditions dans lesquelles elles l'ont fait et les témoignages indirects qui en sont restés ${ }^{16}$ font plutôt croire à un mouvement presque généralisé, non encadré pour ne pas dire spontané - et très largement déterminé par la rareté des terres dans la région-mère, devenue incapable de pourvoir à un croît démographique annuel trop rapide.

\footnotetext{
11 Ces faits sont rapportés par divers auteurs, dont Victor Tremblay (Histoire du Saguenay, 237), qui ne donnent habituellement pas leurs sources mais qui doivent tous à Louis-Antoine Martel, Notes sur le Saguenay. Manuscrit de l'abbé L.-A. Martel, curé de Saint-Alexis, 18581865 (Chicoutimi, Centre d'études et de recherches historiques du Saguenay, 1968), 108 p.

12 Une autre source évoque «les 17 colons» débarqués à la Baie en 1838 (Archives nationales du Québec à Chicoutimi, Mémoires d'Anciens no 446, 2).

${ }_{13}$ Sur tout ce qui précède, Victor Tremblay, Histoire du Saguenay; Raoul Blanchard, L'Est du Canada français «Province de Québec» (Montréal, Librairie Beauchemin Limitée, 1935, tome 2), 336 p.; Léonidas Bélanger, loc. cit.; L.-A. Martel, op. cit. 1839.

${ }_{14}$ Notamment ceux d'un abbé Decoigne (curé de Baie-Saint-Paul?), venu au Saguenay en

is Raoul Blanchard, op. cit., 2: 67.

16 En particulier sous la forme d'une collection d'histoires de vie (Mémoires d'Anciens) conservée aux Archives nationales du Québec à Chicoutimi.
} 


\section{3 - LES PRÉSUMÉS PIONNIERS DU SAGUENAY}

Dans cette direction, notre effort a consisté dans un premier temps à étudier de plus près la contribution des 60 personnes généralement données comme étant à l'origine de la population régionale. La liste de ces présumés pionniers a été constituée à partir de tous les auteurs ayant déjà écrit sur ce sujet (et dont les principaux sont cités plus haut). Dans tous les cas, la contribution de ces défricheurs se serait située aux tout premiers temps de la colonisation, soit en 1838-1840. Ce sont les membres de la Société des Vingt-et-Un, quinze de leurs associés (un 16e a dû être exclu parce qu'il apparaît aussi parmi les précédents), les familles pionnières de l'Anse-Saint-Jean $(\mathrm{N}=6)$ et de la Baie des Hahas $(\mathrm{N}=9)$, une famille de Sainte-Rose et 9 autres personnes dont le nom est mentionné ici et là mais sans référence précise ${ }^{17}$. Pour chacun des individus ou couples listés, nous avons cherché à déterminer a) s'ils avaient véritablement séjourné au Saguenay, b) le cas échéant, pendant quelle durée, $\mathrm{c}$ ) s'ils étaient décédés dans la région, d) le nombre d'enfants mariés qu'ils y ont laissés (ou descendance utile), e) le nombre total de descendants au Saguenay sur quelque 125 ans. Soulignons que, tous ces pionniers ayant été mariés, ce sont en fait des couples dont nous suivons le destin, même si les limites imposées par nos sources nous contraignent parfois à ne suivre que les conjoints (par exemple tableaux 1 et 2).

Cette enquête nous a amenés à mettre à profit l'ensemble des sources nominatives actuellement disponibles, les plus susceptibles d'attester la présence et le déplacement des personnes au début de l'immigration. Les principales sources utilisées sont:

- Les ouvrages déjà mentionnés (Tremblay, Bélanger et autres). Les mentions que nous en avons tirées proviennent d'archives très diverses: rapports de missionnaires, d'inspecteurs ou d'arpenteurs, relations de contemporains, requêtes, minutes notariales, etc.

- Le fichier-réseau de la population BALSAC (pour le Saguenay).

- Les manuscrits des recensements canadiens de 1852, 1861 et 1871.

- Les recensements de l'arpenteur Duberger à la Baie des Hahas en 1842,1845 et $1848^{18}$.

- Le fonds des Mémoires d'Anciens, conservés aux Archives nationales du Québec à Chicoutimi.

17 La liste de ces 60 présumés pionniers du Saguenay est donnée dans le Document no. IIC-138 de SOREP. Il est à noter que le nom de Peter McLeod, né à Chicoutimi vers 1805-1810, n'y figure pas. Nous l'avons associé à l'ère commerciale de la traite des fourrures, pour les fins de cette recherche.

18 Gérard Bouchard et Michel Bergeron, «L'arpenteur J.-B. Duberger et les premiers recensements de la population saguenayenne au XIXe siècle», Archives, 8,3 (décembre 1976): 11-20. 
- Le greffe des arpentages du gouvernement du Québec (ministère de l'Energie et des Ressources).

- Le fonds généalogique et biographique Larouche/Simard, conservé à SOREP.

Il est possible que quelques immigrants isolés nous aient échappé, malgré les nombreux recoupements effectués. Mais il n'y a guère moyen de faire plus dans les conditions actuelles.

La première vérification a porté sur le lieu de sépulture (tableau 1). Sur les 60 candidats, 23 seulement ont été inhumés au Saguenay et y ont donc vraisemblablement vécu jusqu'à leur décès ${ }^{19}$. Il est intéressant de noter que parmi les 36 sociétaires et associés, pas plus de 12 sont venus y terminer leur vie. Quant aux durées de résidence dans la région, il faut d'abord signaler que la présence de 35 pionniers seulement a pu être attestée avec certitude (tableau 2). Les deux tiers d'entre eux ont séjourné 20 ans ou plus. Pour ce qui est des autres, 13 n'ont laissé aucune trace dans nos sources et dans 12 autres cas, il y a incertitude due à des problèmes de jumelage, les données disponibles n'étant pas assez discriminantes ${ }^{20}$. On note par ailleurs, mais sans surprise, que ceux qui sont décédés au Saguenay y ont aussi séjourné en général plus

\section{TABLEAU 1}

Distribution des présumés pionniers selon le lieu de leur inhumation (au Saguenay ou à l'extérieur)

\begin{tabular}{|l|c|c|c|}
\hline \multirow{2}{*}{ Présumés pionniers } & \multicolumn{2}{|c|}{ Lieu de sépulture } & \multirow{2}{*}{ TOTAL } \\
\cline { 2 - 3 } & $\begin{array}{c}\text { Au } \\
\text { Saguenay }\end{array}$ & $\begin{array}{c}\text { Hors } \\
\text { Saguenay }\end{array}$ & \\
\hline Sociétaires & 6 & 15 & 21 \\
(Vingt-et-Un) & 6 & 9 & 15 \\
Associés & 5 & 1 & 6 \\
Colons de l'Anse St-Jean & 4 & 5 & 9 \\
Colons de la Baie & 2 & 7 & 9 \\
Autres & 23 & 37 & 60 \\
\hline \multicolumn{1}{|c|}{ TOTAL } & & & \\
\hline \multicolumn{1}{|c|}{} \\
\hline
\end{tabular}

\footnotetext{
19 Dans le cas des couples, nous avons effectué les compilations en fonction du destin de l'homme.

${ }^{20}$ On ne dispose dans ce cas que d'un nom et d'un prénom, ce qui crée d'insolubles situations d'homonymie.
} 
longtemps que les autres. Enfin, le détail (non reproduit ici) du tableau 2 fait voir que, parmi les 21 sociétaires, 11 seulement ont très certainement séjourné dans la région, d'après les sources qui ont pu être consultées.

TABLEAU 2

Durée de résidence selon le lieu d'inhumation (présumés pionniers du Saguenay)

\begin{tabular}{|c|c|c|c|}
\hline \multirow{2}{*}{$\begin{array}{l}\text { Durée de résidence } \\
\text { (années) } \\
\text { (a) }\end{array}$} & \multicolumn{2}{|c|}{ Lieu de sépulture } & \multirow{2}{*}{ TOTAL } \\
\hline & $\begin{array}{c}\mathrm{Au} \\
\text { Saguenay }\end{array}$ & $\begin{array}{c}\text { Hors } \\
\text { Saguenay }\end{array}$ & \\
\hline 0 & - & 13 & 13 \\
\hline $1-5$ & - & - & - \\
\hline $6-10$ & - & 6 & 6 \\
\hline $11-15$ & 1 & 3 & 4 \\
\hline $16-19$ & 2 & - & 2 \\
\hline 20 et plus & 19 & 4 & 23 \\
\hline Indéterminés & 1 & 11 & 12 \\
\hline TOTAL & 23 & 37 & 60 \\
\hline
\end{tabular}

(a) Les durées de résidence doivent être tenues pour maximales puisque nous n'avons pas tenu compte des migrations temporaires hors de la région. Ces durées ont été fixées à partir de diverses sources nominatives permettant d'établir parfois des mesures précises, parfois des fourchettes seulement. C'est pourquoi nous n'avons pas calculé de durée moyenne.

TABLEAU 3

Descendance utile au Saguenay des présumés pionniers, selon leur lieu d'inhumation

\begin{tabular}{|c|c|c|c|}
\hline \multirow{2}{*}{$\begin{array}{c}\text { Nombre d'enfants } \\
\text { mariés au Saguenay } \\
\text { (a) }\end{array}$} & \multicolumn{2}{|c|}{ Lieu de sépulture } & \multirow{2}{*}{ TOTAL } \\
\cline { 2 - 4 } & $\begin{array}{c}\text { Au } \\
\text { Saguenay }\end{array}$ & $\begin{array}{c}\text { Hors } \\
\text { Saguenay }\end{array}$ \\
\hline 0 & 3 & 25 & 28 \\
$1-2$ & 1 & 10 & 11 \\
$3-4$ & 3 & - & 5 \\
$5-6$ & 11 & - & 4 \\
$7-8$ & 4 & - & 1 \\
9 et plus & 1 & 37 & 60 \\
\hline TOTAL & 23 & - & 4 \\
\hline
\end{tabular}

(a) Quelques enfants ont contracté mariage hors du Saguenay et sont revenus s'y établir ensuite; ils n'ont pas été inclus dans ce calcul.

Source: Fichier BALSAC (population du Saguenay). 
Une autre indication - directe celle-là - de la contribution à la formation d'une population est donnée par la mesure de la descendance, et d'abord la descendance utile, c'est-à-dire le nombre d'enfants éventuellement mariés issus d'un couple. Sous ce rapport, le tableau 3 fait ressortir la très grande inégalité des apports individuels. Près de la moitié des individus en effet n'ont eu aucune descendance utile au Saguenay; d'un autre côté, cinq pionniers rendent compte pratiquement à eux seuls du tiers des mariages d'enfants (40 sur 131) de la seconde génération.

Le fichier de population a permis d'aller plus loin et de calculer la descendance des mêmes individus sur six générations. Les résultats sont présentés au tableau 4. Ce dernier montre que le quart des présumés pionniers n'ont pas enregistré de naissance au Saguenay, que plus du tiers $(\mathrm{N}=22)$ n'y sont plus représentés après cinq générations et que cinq ancêtres ont assuré une part exceptionnelle dans la reproduction de la population. Par ailleurs, ces chiffres de descendance doivent être interprétés avec prudence, à la lumière des notes de méthode regroupées au bas du tableau 4 .

Toutes les données qui précèdent concernent le groupe des présumés pionniers, selon l'historiographie régionale la mieux accréditée. Ces reconstitutions auxquelles nous nous sommes livrés visaient, dans un esprit critique, à évaluer la véritable contribution de ce groupe à la naissance de la nouvelle région. Il en ressort que de $22 \%$ à $42 \%$ de ces ancêtres n'ont jamais résidé au Saguenay (ceux-là méritent tout au plus le titre de promoteurs), que la moitié seulement y ont certainement vécu plus de dix ans, ce qui leur donne droit au statut de pionniers, et que près de la moitié d'entre eux n'y ont pas eu de descendance utile, ce qui les exclut du rang des ancêtres et des fondateurs, tels que définis ici.

Nous pensons que, pour l'histoire régionale en général, il y a des leçons importantes à tirer de ces conclusions. En effet, on voit qu'il peut exister entre l'historiographie et la mémoire populaire des promiscuités gênantes, qui ne portent profit ni à l'une ni à l'autre. C'est au moins un cas où la pratique scientifique historienne a intérêt à se démarquer du quotidien et de ses actualités. Mais elle ne peut le faire que si elle se donne les moyens d'observation appropriés. Qu'il s'agisse du Saguenay ou de toute autre région du Québec, il est certain qu'on connaît trop peu de choses sur la naissance des populations locales ou régionales, sur les modalités de leur croissance et de leur reproduction. De ce point de vue, les fichiers de population constituent des instruments privilégiés, en particulier lorsqu'ils recouvrent des régions ou des pays neufs. 


\section{TABLEAU 4}

Distribution des 60 présumés pionniers selon le nombre de leurs descendants nés au Saguenay, à diverses générations. (Premières unions seulement).

Deuxième génération (a)

\begin{tabular}{|c|c|c|c|c|c|c|}
\hline $\begin{array}{c}\text { N. de descendants } \\
\text { (b) }\end{array}$ & 0 & $1-4$ & $5-6$ & $7-10$ & $11-14$ & $15-18$ \\
\hline $\begin{array}{c}\text { Distribution des } \\
\text { présumés pionniers }\end{array}$ & 15 & 15 & 9 & 16 & 3 & 2 \\
\hline
\end{tabular}

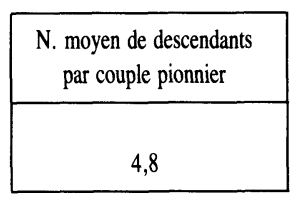

Troisième génération

\begin{tabular}{|c|c|c|c|c|c|c|}
\hline N. de descendants & 0 & $1-10$ & $11-20$ & $21-40$ & $41-80$ & $81-160$ \\
\hline $\begin{array}{c}\text { Distribution des } \\
\text { présumés pionniers }\end{array}$ & 18 & 3 & 8 & 7 & 20 & 4 \\
\hline
\end{tabular}

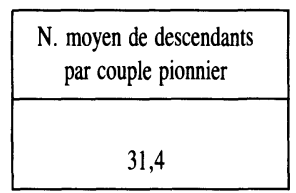

Quatrième génération

\begin{tabular}{|c|c|c|c|c|c|c|}
\hline N. de descendants & 0 & $1-100$ & $101-200$ & $201-300$ & $301-400$ & $401-700$ \\
\hline $\begin{array}{c}\text { Distribution des } \\
\text { présumés pionniers }\end{array}$ & 21 & 12 & 12 & 10 & 1 & 4 \\
\hline
\end{tabular}

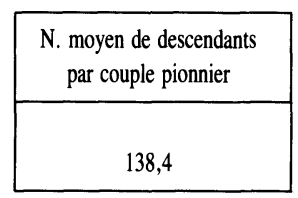

\section{Cinquième génération}

\begin{tabular}{|c|c|c|c|c|c|c|c|}
\hline N. de descendants & 0 & $1-50$ & $51-100$ & $101-500$ & $501-1000$ & $\begin{array}{l}1001- \\
3000\end{array}$ & $\begin{array}{l}\text { N. moyen de descendants } \\
\text { par couple pionnier }\end{array}$ \\
\hline $\begin{array}{c}\text { Distribution des } \\
\text { présumés pionniers }\end{array}$ & 22 & - & 2 & 19 & 12 & 5 & 431,7 \\
\hline
\end{tabular}

\section{Sixième génération}

\begin{tabular}{|c|c|c|c|c|c|c|}
\hline $\begin{array}{c}\text { N. de descendants } \\
\text { (c) }\end{array}$ & 0 & $1-50$ & $51-100$ & $101-1000$ & $\begin{array}{c}1001- \\
3000\end{array}$ & $\begin{array}{c}3001- \\
7000\end{array}$ \\
\hline $\begin{array}{c}\text { Distribution des } \\
\text { présumés pionniers }\end{array}$ & 22 & 2 & 1 & 17 & 13 & 5 \\
\hline
\end{tabular}

Notes: (a) La première génération est celle des présumés pionniers eux-mêmes.

(b) Pour le calcul de cette génération, nous avons inclus parmi les descendants les fils et filles de pionniers nés et mariés hors Saguenay et ayant immigré avec leurs parents $(\mathrm{N}=91)$. Mais ce travail n'a pu être fait pour les générations subséquentes, ce qui est une cause de sous-évaluation des descendances.

(c) À l'intérieur d'une même descendance, s'il y a mariage entre consanguins de même génération ou non, les enfants ne sont comptés qu'une fois. Mais s'il y a mariage entre descendants de deux pionniers différents, les enfants sont comptés deux fois. Les descendances ne peuvent donc pas être additionnées. 
Cet exercice a été fructueux sur un second plan, dans la mesure où il a fourni un aperçu des contributions très inégales des ancêtres à la formation de la population régionale. Pour cette raison, il a paru utile de le prolonger en lui donnant une portée plus étendue et en partant d'une population de pionniers plus rigoureusement définie.

\section{4 - FORMATION ET REPRODUCTION DE LA POPULATION SAGUENAYENNE}

Pour obtenir une image plus exacte de la réalité, nous avons redéfini la sous-population des pionniers, celle-ci englobant cette fois tous les individus dont la résidence est dûment attestée dans la région entre 1838 et 1841 inclusivement. Rappelons que l'année 1842 coïncide avec la création de la première paroisse (Saint-Alexis-de-Grande-Baie); la période pionnière est ainsi caractérisée par l'absence de toute institution. Notons aussi que la notion de pionniers renvoie ici à l'échelle régionale, étant entendu qu'elle peut se prêter ultérieurement à des acceptions plus restreintes, notamment dans le cadre de la création de paroisses. L'objectif de l'enquête est ici de recenser tous les contributeurs potentiels à la nouvelle population régionale et de mesurer leur apport effectif sur six générations afin d'esquisser un modèle d'analyse - et non un palmarès des pionniers les plus méritants.

À l'aide de trois sources différentes ${ }^{21}$, il a été possible d'identifier 431 hommes et femmes ayant résidé au Saguenay avant 1842 et s'étant mariés dans la région ou dans Charlevoix. Nous excluons ainsi les célibataires définitifs (en nombre négligeable) et les pionniers ayant ultérieurement émigré puis contracté mariage hors du Saguenay (ils sont extrêmement difficiles à identifier et leur nombre est impossible à établir exactement). Le taux d'enracinement de ces pionniers est beaucoup plus fort que celui du groupe étudié précédemment. Plus de 7 sur 10 $(72,2 \%)$ sont décédés dans la région et une proportion presque semblable $(68,9 \%)$ y a vécu 20 ans et plus (tableau 5). On observe une différence du même genre au chapitre de la descendance utile (tableau 6). Près de la moitié des couples représentés parmi ces pionniers ont marié cinq enfants et plus au Saguenay, proportion qui dépasse $60 \%$ dans le cas des pionniers décédés sur place. Deux éléments sont à retenir de ces données: la très forte contribution de ces pionniers à la mise en place de la première génération de Saguenayens et la grande inégalité qui se dégage, encore une fois, autant pour la descendance utile que pour les durées de séjour. Soulignons en effet que $54,7 \%$ des enfants mariés à la deuxième génération sont issus de $28,3 \%$ seulement des pionniers. Mais à l'autre bout de la distribution, il est non moins vrai que $32,5 \%$ des pionniers n'assurent que $5,2 \%$ de toute la descendance utile.

21 Il s'agit des registres de mariages de Charlevoix, le fichier BALSAC (pour le Saguenay) et un repêchage parmi les 60 présumés pionniers étudiés plus haut. 
TABLEAU 5

Durée de résidence des 431 pionniers (a) selon le lieu de sépulture. Saguenay.

\begin{tabular}{|c|r|r|r|r|r|r|}
\hline \multirow{2}{*}{$\begin{array}{c}\text { Durée de résidence } \\
\text { (années) }\end{array}$} & \multicolumn{3}{|c|}{ Lieu de sépulture } & \multicolumn{2}{c|}{ TOTAL } \\
\cline { 2 - 6 } & \multicolumn{2}{|c|}{$\begin{array}{c}\text { Au } \\
\text { Saguenay }\end{array}$} & \multicolumn{2}{c|}{$\begin{array}{c}\text { Hors } \\
\text { Saguenay }\end{array}$} & \multicolumn{2}{c|}{} \\
\hline & N. a. & \multicolumn{1}{|c|}{$\%$} & N. a. & $\%$ & N. a. & $\%$ \\
\hline $1-5$ (b) & 28 & 9,0 & 30 & 29,4 & 58 & 14,0 \\
$6-10$ & 11 & 3,5 & 16 & 15,7 & 27 & 6,5 \\
$11-15$ & 17 & 5,5 & 12 & 11,8 & 29 & 7,0 \\
$16-19$ & 12 & 3,9 & 3 & 2,9 & 15 & 3,6 \\
20 et plus & 243 & 78,1 & 41 & 40,2 & 284 & 68,9 \\
\hline TOTAL & 311 & 100,0 & 102 & 100,0 & 413 & 100,0 \\
\hline Indéterminés & - & - & 18 & - & 18 & - \\
& & & (c) & & & \\
\hline
\end{tabular}

Notes: a) Personne dont la résidence a pu être attestée dans la région avant 1842 .

b) Il n'y a pas de catégorie 0 puisque, par définition, le statut de pionnier implique résidence. Les séjours de quelques mois ont été classés avec ceux d'un an. Voir aussi la note (a) accompagnant le tableau 2.

c) À cause des problèmes de jumelage posés par le recensement de Duberger (1844), il a été impossible de déterminer la durée de séjour de ces 18 personnes.

\section{TABLEAU 6}

Descendance utile au Saguenay des 431 pionniers, selon leur lieu de sépulture.

\begin{tabular}{|c|r|r|r|r|r|r|}
\hline \multirow{2}{*}{$\begin{array}{c}\text { Nombre d'enfants } \\
\text { mariés au Saguenay }\end{array}$} & \multicolumn{3}{|c|}{ Lieu de sépulture } & \multicolumn{2}{c|}{ TOTAL } \\
\cline { 2 - 5 } & \multicolumn{2}{|c|}{$\begin{array}{c}\text { Au } \\
\text { Saguenay }\end{array}$} & \multicolumn{2}{c|}{$\begin{array}{c}\text { Hors } \\
\text { Saguenay }\end{array}$} & \multicolumn{2}{c|}{} \\
\hline & N. a. & \multicolumn{1}{|c|}{$\%$} & N. a. & $\%$ & N. a. & $\%$ \\
\hline 0 & 17 & 5,5 & 57 & 47,5 & 74 & 17,2 \\
$1-2$ & 36 & 11,6 & 30 & 25,0 & 66 & 15,3 \\
$3-4$ & 65 & 20,9 & 14 & 11,6 & 79 & 18,3 \\
$5-6$ & 83 & 26,7 & 7 & 5,8 & 90 & 20,9 \\
$7-8$ & 76 & 24,4 & 7 & 5,8 & 83 & 19,3 \\
9 et plus & 34 & 10,9 & 5 & 4,3 & 39 & 9,0 \\
\hline TOTAL & 311 & 100,0 & 120 & 100,0 & 431 & 100,0 \\
\hline
\end{tabular}


Nous avons également calculé la descendance de ces couples pionniers jusqu'à la sixième génération (tableau 7) ${ }^{22}$. On note d'abord le nombre élevé de descendants à la dernière génération, la moyenne s'établissant à 1032 pour chaque couple pionnier. On relève aussi une très grande inégalité dans la contribution des fondateurs, dont $22,1 \%$ ne sont même plus représentés dans la population actuelle. À l'opposé, une minorité d'entre eux enregistrent des performances très supérieures à la moyenne, tel le couple Jean-Baptiste Côté/Émérance Girard, avec 9267 descendants (soit environ 3\% de la population régionale). Ces écarts considérables entre les pionniers ne sont évidemment pas dus uniquement à des différences dans la fécondité comme telle. La mortalité des enfants y jouait un grand rôle, et plus encore sans doute l'émigration. Rappelons qu'entre 1852 et 1861 seulement, le quart des familles saguenayennes quittent la région ${ }^{23}$. En outre, des 1036 couples formés au Saguenay entre 1842 et 1861, environ le tiers n'y laissent aucune descendance, dans la plupart des cas parce qu'ils émigrent avant la naissance d'un premier enfant ${ }^{24}$. Une mesure diachronique du prélèvement migratoire est présentée au tableau 8. Pour la période 18421881 , ce dernier donne par sous-période quinquennale la proportion des individus nés dans la région et ayant ultérieurement émigré ${ }^{25}$. Cette proportion avoisine la moitié et varie peu durant toute la période, ce qui révèle à la fois les difficultés d'enracinement des familles immigrantes et le problème souvent insurmontable que représentait pour les familles déjà implantées l'établissement de tous leurs enfants. Ces données éclairent aussi un aspect important de la dynamique de la colonisation, laquelle se nourrissait comme on voit d'une part importante d'échecs ${ }^{26}$. À la longue cependant, l'écoumène s'étend, l'urbanisation prend forme et la région retient de mieux en mieux son croît démogra-

22 En pratique, le fichier de population aurait permis de poursuivre la construction de plusieurs descendances jusqu'à la septième génération. Nous ne l'avons pas fait car un certain nombre de lignées se seraient ainsi trouvées amputées, faute d'une période d'observation suffisamment longue («truncation bias»). Il est vraisemblable d'ailleurs que, même à la 6e génération, le nombre des descendants est sous-estimé.

${ }_{23}$ Christian Pouyez, Raymond Roy et Gérard Bouchard, «La mobilité géographique en milieu rural. Le Saguenay, 1852-1861», Histoire sociale/Social History, 14,27 (mai 1981): 123155.

24 Données compilées par R. Roy, soumises pour publication.

25 En fait, pour chaque naissance relevée dans le fichier entre 1842 et 1881 , nous avons recherché un décès correspondant dans un intervalle de 90 ans. L'absence d'un décès était alors identifiée à une émigration.

26 À titre de comparaison, notons qu'entre 1621 et 1740 , la proportion des enfants nés dans la ville de Québec et décédés à l'extérieur s'établit aux environs des deux tiers (Danielle Gauvreau, Reproduction humaine et reproduction sociale: la ville de Québec pendant le Régime français, thèse de doctorat présentée à la Faculté des études supérieures, département de démographie, Université de Montréal, collection de thèses et mémoires, no 13, chapitre 9, 1986, 442 p.) La région de colonisation retenait davantage sa population que la ville pionnière. Notons par ailleurs que dans le cas de Québec, le sous-enregistrement des sépultures d'enfants explique une partie non négligeable du phénomène. Mais ce n'est pas le cas au Saguenay où, d'après les sondages réalisés jusqu'ici, ce sous-enregistrement était faible. 
TABLEAU 7

Répartition des 181 couples pionniers (1ère génération) selon leur nombre de descendants du Saguenay, à diverses générations (a).

(Premiers mariages seulement)

\section{Deuxième génération}

\begin{tabular}{|c|c|c|c|c|c|c|c|c|c|c|c|c|c|c|c|c|}
\hline N. de descendants & & & & $\begin{array}{l}1-4 \\
\text { a. } \%\end{array}$ & & $\begin{array}{l}5-6 \\
\text { a. } \%\end{array}$ & & & & $\begin{array}{r}-14 \\
\%\end{array}$ & & & $\begin{array}{c}\mathrm{T} \\
\text { N.a. }\end{array}$ & & $\begin{array}{l}\mathrm{Nb} \text {. total de des- } \\
\text { cendants } \\
\text { (b) }\end{array}$ & $\begin{array}{l}\mathrm{Nb} \text {. moyen de } \\
\text { desc. par couple } \\
\text { de pionniers }\end{array}$ \\
\hline $\mathrm{N}$. de pionniers & 12 & 6,6 & 41 & 22,6 & 22 & 12,3 & 56 & 30,9 & 37 & 20,4 & 13 & 7,2 & 181 & 100 & 1451 & 8,0 \\
\hline
\end{tabular}

Troisième génération

\begin{tabular}{|c|c|c|c|c|c|c|c|c|c|c|c|c|c|c|c|c|}
\hline N. de descendants & & 0 & & & & $\begin{array}{r}-20 \\
\%\end{array}$ & & $\begin{array}{r}-40 \\
\%\end{array}$ & & $\begin{array}{r}-80 \\
\%\end{array}$ & $\begin{array}{r}\text { Plus } \\
8 \\
\text { N.a. }\end{array}$ & & & & $\begin{array}{l}\text { Nb. total de des- } \\
\text { cendants }\end{array}$ & $\begin{array}{l}\mathrm{Nb} \text {. moyen de } \\
\text { desc. par couple } \\
\text { de pionniers }\end{array}$ \\
\hline $\mathrm{N}$. de pionniers & 29 & 16,0 & 11 & 6,2 & 20 & 11,0 & 29 & 16,0 & 74 & 40,9 & 18 & 9,9 & 181 & 100 & 7389 & 40,8 \\
\hline
\end{tabular}

Quatrième génération

\begin{tabular}{|c|c|c|c|c|c|c|c|c|c|c|c|c|c|c|c|c|}
\hline N. de descendants & & $\begin{array}{c}0 \\
\text { a. } \%\end{array}$ & & $\begin{array}{r}-100 \\
\% . \%\end{array}$ & & $\begin{array}{l}1-200 \\
\text { a. } \%\end{array}$ & & $\begin{array}{r}-300 \\
\%\end{array}$ & & & $\begin{array}{r}\text { Plu } \\
4 \\
\text { N.a. }\end{array}$ & & $\begin{array}{l}\text { To } \\
\text { N.a. }\end{array}$ & & $\begin{array}{c}\mathrm{Nb} \text {. total de des- } \\
\text { cendants }\end{array}$ & $\begin{array}{l}\mathrm{Nb} \text {. moyen de } \\
\text { desc. par couple } \\
\text { de pionniers }\end{array}$ \\
\hline N. de pionniers & 34 & 18,8 & 46 & 25,4 & 44 & 24,3 & 33 & 18,2 & 13 & 7,2 & 11 & 6,1 & 181 & 100 & 27674 & 152,9 \\
\hline
\end{tabular}

Cinquième génération

\begin{tabular}{|c|c|c|c|c|c|c|c|c|c|c|c|c|c|c|c|c|}
\hline N. de descendants & & $\begin{array}{c}0 \\
\text {.a. } \%\end{array}$ & $\begin{array}{r}1 \\
\text { N.a }\end{array}$ & $\begin{array}{l}50 \\
\%\end{array}$ & $\begin{array}{l}51- \\
\text { N.a. }\end{array}$ & $\begin{array}{r}100 \\
\%\end{array}$ & & $\begin{array}{r}1-500 \\
\%\end{array}$ & & $\begin{array}{r}-1000 \\
\%\end{array}$ & & $\begin{array}{l}1 \mathrm{ds} \text { de } \\
000 \\
\%\end{array}$ & & & $\begin{array}{c}\mathrm{Nb} \text {. total de des- } \\
\text { cendants }\end{array}$ & $\begin{array}{l}\mathrm{Nb} \text {. moyen de } \\
\text { desc. par couple } \\
\text { de pionniers }\end{array}$ \\
\hline N. de pionniers & 38 & 21,0 & 4 & 2,2 & 10 & 5,5 & 56 & 30,9 & 47 & 26,0 & 26 & 14,4 & 181 & 100 & 97095 & 536,4 \\
\hline
\end{tabular}

\section{Sixième génération}

\begin{tabular}{|c|c|c|c|c|c|c|c|c|c|c|c|c|c|c|c|c|}
\hline N. de descendants & & $\begin{array}{l}0 \\
\text { a. } \%\end{array}$ & & & $\begin{array}{r}51 \\
\text { N.a }\end{array}$ & $\begin{array}{r}100 \\
\%\end{array}$ & & $\begin{array}{r}-1000 \\
\%\end{array}$ & & $\begin{array}{l}001- \\
\%\end{array}$ & & & & & $\begin{array}{c}\mathrm{Nb} \text {. total de des- } \\
\text { cendants }\end{array}$ & $\begin{array}{l}\mathrm{Nb} \text {. moyen de } \\
\text { desc. par couple } \\
\text { de pionniers }\end{array}$ \\
\hline $\mathrm{N}$. de pionniers & 40 & 22,1 & 5 & 2,8 & 4 & 2,2 & 75 & 41,4 & 40 & 22,1 & 17 & 9,4 & 181 & 100 & 186824 & 1032,2 \\
\hline
\end{tabular}

(a) Couples pionniers = ayant résidé au Saguenay avant 1842. Ces couples constituent la lère génération. L'exclusion des remariages ainsi que des pionniers mariés ayant immigré avec leurs parents ramène à 181 le nombre de couples fondateurs ici pris en compte.

(b) La note (c) du Tableau 4 s'applique ici également. 
TABLEAU 8

Proportion d'émigrants parmi les individus nés au Saguenay entre 1842 et 1881.

\begin{tabular}{|c|c|c|c|}
\hline \multirow{2}{*}{ Sous-périodes } & \multirow{2}{*}{ Naissances } & \multicolumn{2}{|c|}{$\begin{array}{c}\text { Ayant émigré } \\
\end{array}$} \\
\cline { 2 - 4 } & & N. a. & $\%$ \\
\hline $1842-46$ & 569 & 285 & 50,1 \\
$1847-51$ & 1503 & 671 & 44,6 \\
$1852-56$ & 1850 & 849 & 45,9 \\
$1857-61$ & 2422 & 1119 & 46,2 \\
$1862-66$ & 3252 & 1495 & 46,0 \\
$1867-71$ & 4040 & 1947 & 48,2 \\
$1872-76$ & 4865 & 2165 & 44,5 \\
$1877-81$ & 5464 & 2394 & 43,8 \\
\hline TOTAL & 23965 & 10925 & 45,6 \\
\hline
\end{tabular}

(a) Ont été comptés comme émigrants les individus nés au Saguenay et décédés à l'extérieur de la région. Nous nous sommes assurés d'une période minimale d'observation de 90 ans après chaque naissance.

\section{TABLEAU 9}

Distribution décennale des enfants de 1 à 10 ans décédés au Saguenay, selon leur lieu de naissance (à l'intérieur ou à l'extérieur de la région).

\begin{tabular}{|c|c|c|}
\hline $\begin{array}{c}\text { Années de } \\
\text { naissance }\end{array}$ & $\begin{array}{c}\text { N. total } \\
\text { de décès }\end{array}$ & $\begin{array}{c}\text { Nés au } \\
\text { Saguenay } \\
(\%)\end{array}$ \\
\hline $1852-1861$ & 357 (a) & 76,8 \\
$1862-1871$ & 621 & 79,7 \\
$1872-1881$ & 720 & 83,6 \\
$1882-1891$ & 1165 & 84,7 \\
$1892-1901$ & 1373 & 84,4 \\
$1902-1911$ & 1399 & 85,2 \\
$1912-1921$ & 1697 & 86,0 \\
\hline
\end{tabular}

(a) Les registres saguenayens de l'état civil s'ouvrent en 1842 .

phique, aussi bien naturel que migratoire. C'est ce que montre l'évolution décennale de la proportion des enfants nés au Saguenay parmi ceux qui y sont décédés entre 1 et 10 ans (tableau 9).

Pour le reste, il va de soi que les données présentées ici demeurent préliminaires. Pour mieux rendre compte de la dynamique de la repro- 
duction inégale, il faudra conduire des analyses plus fines destinées à préciser le rôle respectif de la fécondité et de la mortalité différencielles, de la nuptialité et de l'émigration.

\section{5 - MYTHES ET RÉALITÉ DE LA REPRODUCTION}

Si forts soient-ils, les écarts de reproduction observés parmi les couples pionniers ne constituent pas un phénomène exclusif au Saguenay. Des écarts analogues ont été relevés dans des populations très diverses, qu'il s'agisse de la célèbre tribu des Touareg Kel Kummer ${ }^{27}$, des Amish ${ }^{28}$, de la population danoise ${ }^{29}$ ou des pionniers de la Nouvelle-France ${ }^{30}$. Par ailleurs, on sait que le niveau général de la fécondité saguenayenne semble particulièrement élevé pour la période ${ }^{31}$. Ainsi, $19,3 \%$ des couples pionniers de la Nouvelle-France au XVIIe siècle ont eu une descendance utile (c'est-à-dire un nombre moyen de fils et de filles ultérieurement mariés) de 7 enfants et plus, comparativement à $28,3 \%$ pour les pionniers saguenayens ${ }^{32}$. Ces données attirent encore une fois l'attention sur les niveaux très élevés de la fécondité en contexte de peuplement.

Au Saguenay comme ailleurs, la tradition orale a accrédité l'idée que les naissances survenaient à un rythme annuel dans ce genre de population, caractérisée par un régime de fécondité dite naturelle. Comme on sait, la recherche en démographie historique a fait justice de cette représentation en montrant que l'intervalle intergénésique moyen, durant la période fertile de la vie d'un couple, avoisinait en général deux années plutôt qu'une. Même en ne considérant que les familles les plus fécondes, le cliché populaire ne se vérifie pas. Ainsi, nous avons pu extraire de notre fichier de population 23 familles ayant enregistré 20 naissances et plus (le maximum est de 22) en autant de grossesses, ce qui exclut donc les jumeaux et triplets. Dans ces conditions exceptionnelles, l'intervalle intergénésique moyen n'est pas inférieur à 16 mois $^{33}$. Mais malgré ces démentis, il reste que la tradition

\footnotetext{
27 Albert Jacquard, «Évolution du patrimoine génétique des Kel Kummer», Population, 4-5 (juillet-octobre 1972): 784-800.

${ }_{28}$ Victor A. McKusick et al., Medical Genetic Studies of the Amish (Baltimore, The Johns Hopkins University Press Ltd, 1983), 519 p.

${ }_{29}$ M. Lamy et J. De Grouchy, L'Homme et l' hérédité (Paris, Hachette, 1967), 281 p.

30 Hubert Charbonneau, André Guillemette et al., Naissance d'une population. Les Français établis au Canada au XVIIIe siècle (Paris/Montréal, INED/Presses de l'Université de Montréal, coll. «Travaux et Documents», no 118, 1987), 232 p.

31 Christian Pouyez, Yolande Lavoie, Gérard Bouchard, Raymond Roy, et al., loc. cit.; Gérard Bouchard, «Sur la reproduction familiale en milieu rural: systèmes ouverts et systèmes clos", Recherches sociographiques, 28,2-3 (1987): 229-251.

32 Une partie de cet écart est sans doute imputable à des différences dans le degré d'enracinement, le taux de célibat et la mortalité en bas âge, entre les deux populations.

33 Ces femmes ont donc vécu 20 fois et plus, sans interruption, le cycle: 9 mois de grossesse / 1 mois de «relevailles» / 5-6 mois d'allaitement. Soit au total, sur 27 années de vie fertile en moyenne, l'équivalent de 16 années environ en état de grossesse...
} 
orale n'est pas tout à fait sans fondement. Les intervalles dont nous venons de faire état ne sont que des moyennes qui effacent les changements de rythme caractérisant le calendrier des naissances - on sait par exemple que les intervalles réels s'allongent vers la fin du calendrier. En ne retenant cette fois que les naissances survenant entre 20 ans et le début de la trentaine, on obtient un intervalle moyen de 13 mois. La représentation courante a donc visiblement retenu les rythmes de procréation qui correspondent à la période la plus active de la vie féconde des mères. Cet exemple illustre la façon dont une même réalité, diversement appréhendée, peut accréditer deux représentations différentes. Mais la première seulement relève d'une démarche scientifique, parce que son mode de construction est contrôlé et vérifiable.

Une dernière dimension associée à la reproduction et aux descendances concerne la diffusion des patronymes. C'est là, encore une fois, un objet culturel qui est la proie de clichés parfois très fantaisistes. Ici, il faut évidemment dénoncer en premier lieu la mythologie de «l'ancêtre» direct, qui fait fi des lois élémentaires de la génétique (on sait que l'homme seul transmet le nom aux enfants mais que leurs gènes sont transmis à parts égales par les deux conjoints). En deuxième lieu, les perceptions de l'onomastique, du moins pour ce qui concerne le Saguenay, sont très loin de la réalité. Ainsi, au lieu des $20 \%, 40 \%$ et même $50 \%$ qu'on leur prête assez couramment, les Tremblay n'ont jamais représenté plus de $12 \%$ de la population régionale ${ }^{34}$. En outre, on attribue la fréquence des noms les plus courants dans la population actuelle au fait que les ancêtres qui les ont introduits au milieu du XIXe siècle auraient eu une fécondité plus élevée et une descendance plus nombreuse que les autres. Nous avons pu établir qu'au contraire, ces familles se sont reproduites moins vite que les autres et leur descendance est inférieure à la moyenne (tableau 10$)^{35}$. La fréquence relative des Tremblay, par exemple, a été réduite du tiers depuis le XIXe siècle. En fait, ces patronymes doivent leur fréquence actuelle tout simplement

34 Gérard Bouchard, Marie-Ange Desjardins-Ouellette, France Markowski et al., «La distribution des patronymes au Québec: témoins des dynamiques de population», Anthropologie et Sociétés, 9,3 (1985): 197-218. Gérard Bouchard, Jean Morissette, Kevork Kouladjian, «La statistique agrégée des patronymes du Saguenay et de Charlevoix comme indicateurs de la structure de la population aux XIXe et XXe siècles», Cahiers québécois de démographie, 16,1 (avril 1987): 67-98.

35 Parmi les 10 noms de famille qui se sont reproduits le plus rapidement depuis 1838 , un seul compte parmi les 15 noms les plus fréquents aujourd'hui. Par ailleurs, dans la mesure où la reproduction d'un segment de la population résulte d'un ensemble de facteurs comme la fécondité, la mortalité, la nuptialité (incluant les remariages) et l'émigration, il est légitime de considérer que la fréquence d'un patronyme reflète la vitesse de reproduction des descendances qui le portent. Certes, ce postulat peut entraîner quelques biais du fait que seuls les hommes transmettent les patronymes. Mais dans une population assez nombreuse comme celle du Saguenay, et sur une période assez longue, ces biais ne sont pas de nature à invalider l'observation de tendances. 


\section{TABLEAU 10}

Descendance des pionniers saguenayens ayant eu des descendants jusqu'à la 6e génération, selon la fréquence de leur patronyme.

\begin{tabular}{|c|c|c|c|c|}
\hline \multirow[b]{2}{*}{ Patronymes } & \multicolumn{2}{|c|}{$\begin{array}{l}\text { Fréquence dans la population } \\
\qquad(1962-1971)\end{array}$} & \multicolumn{2}{|c|}{ Descendance à la $6 e$ génération } \\
\hline & $\begin{array}{c}\text { Fréquence } \\
\text { relative } \\
(\%)\end{array}$ & $\begin{array}{c}\text { Rang parmi } \\
\text { l'ensemble des } \\
\text { patronymes }\end{array}$ & $\begin{array}{c}\text { Nombre } \\
\text { moyen } \\
\text { de descen- } \\
\text { dants }\end{array}$ & $\begin{array}{c}\text { Rang parmi } \\
\text { l'ensemble } \\
\text { des patronymes } \\
(\mathrm{N}=54)\end{array}$ \\
\hline TREMBLAY & 8,18 & 1 & 1071 & 24 \\
\hline GAGNON & 3,17 & 2 & 1174 & 19 \\
\hline BOUCHARD & 3,11 & 3 & 1042 & 25 \\
\hline SIMARD & 2,89 & 4 & 1291 & 16 \\
\hline GIRARD & 2,52 & 5 & 1114 & 23 \\
\hline LAVOIE & 2,15 & 6 & 1149 & 20 \\
\hline FORTIN & 2,12 & 7 & 430 & 43 \\
\hline CÔTÉ & 1,82 & 8 & 5509 & 4 \\
\hline GAUTHIER & 1,68 & 9 & 1014 & 29 \\
\hline LAROUCHE & 1,49 & 10 & 375 & 45 \\
\hline GAGNÉ & 1,37 & 11 & 1490 & 15 \\
\hline BERGERON & 1,33 & 12 & 1007 & 30 \\
\hline SAVARD & 1,17 & 13 & 99 & 52 \\
\hline HARVEY & 1,04 & 14 & 1015 & 26 \\
\hline BOIVIN & 1,02 & 15 & 791 & 35 \\
\hline
\end{tabular}

- Nombre moyen de descendants pour l'ensemble des pionniers: 1320.

- Nombre de pionniers considérés: 141 .

- Les femmes ont dû être exclues du fait qu'elles ne transmettaient pas leur patronyme.

au fait qu'ils étaient plus nombreux au départ, parmi les premiers immigrants défricheurs ${ }^{36}$.

\section{CONCLUSION}

À la lumière des quelques exemples présentés ici, il est permis en premier lieu de suggérer que la mémoire collective régionale est mauvaise historienne. Elle sélectionne, grossit ou déforme selon des critères et des procédés qui ne sont manifestement pas ceux de la profession. En particulier, elle semble se caractériser par un esprit critique très inégal et une certaine complaisance dans des représentations simplistes,

\footnotetext{
${ }^{36}$ Ainsi, on comptait $13,7 \%$ de Tremblay parmi les pionniers attestés avant 1842 , une proportion qui n'a cessé de diminuer par la suite.
} 
définitivement accréditées. Par rapport à la science historique au sens strict, elle relève d'un discours parallèle, sinon concurrent, mais tout aussi fonctionnel, qui s'alimente et se diffuse selon des voies qui lui sont propres. Considérée à l'échelle régionale, elle offre une image radicale de l'opposition entre ce qu'il est convenu d'appeler la culture savante et la culture populaire; mais une culture populaire réorganisée, qui s'est donné des lettres, des titres et des canaux de diffusion formalisés.

En deuxième lieu, on pourrait être tenté d'utiliser nos données sur les descendances pour corroborer un autre cliché tenace, selon lequel la plupart des Saguenayens descendraient d'un nombre très restreint peut-être quelques dizaines - d'immigrants pionniers ou fondateurs. Il est donc prudent de rappeler que nous avons ici limité l'observation aux pionniers établis avant 1842 . Or, nous savons que l'immigration régionale s'est poursuivie et amplifiée par la suite, tout au long du XIXe et du XXe siècle ${ }^{37}$. Il faut voir la formation de la population saguenayenne non pas comme un phénomène ponctuel, enfermé dans deux ou trois décennies, mais comme un processus séculaire de renouvellement migratoire, d'accroissement démographique et d'expansion territoriale, le tout ponctué de reculs et de reprises.

Enfin, en ce qui concerne la contribution réelle de ces ancêtres immigrants à la constitution du bassin génétique régional, il faut se garder encore là d'inférences apparemment raisonnables et néanmoins sans fondement. En effet, les aléas des migrations et de la transmission génétique déjouent parfois les données les plus fiables. D'abord, ce ne sont pas toujours les ancêtres qui se sont le plus reproduits qui ont le plus de descendants dans la population actuelle. En outre, et surtout, les généticiens ont bien montré que ce ne sont pas nécessairement les ancêtres ayant le plus de descendants aujourd'hui qui ont le plus contribué au pool génique ${ }^{38}$.

Pour toutes ces raisons, à chaque fois que l'attention se porte sur l'origine d'une région ou d'une population, il est nécessaire de bien définir les phénomènes à reconstituer et les dynamiques spécifiques qui leur sont associées: animation et organisation, immigration, création d'établissements, reproduction démographique, transmission génétique. D'où la pertinence des notions de promoteurs, d'immigrants, de pionniers, d'ancêtres et de fondateurs, proposées au début de cette étude.

\footnotetext{
37 Le rythme décennal s'accélère à partir de 1861: 2957 en 1856-1861, 3761 en 18621871,4569 en 1892-1901, 6459 en 1902-1911, etc, D. Gauvreau, loc. cit.

38 Le cas le plus spectaculaire à cet égard est sans doute celui des Kel Kummer, voir A. Jacquard, loc. cit.; parmi les 156 ancêtres de cette tribu au XVIIe siècle, 25 seulement ont apporté une part non négligeable au patrimoine génique récent.
} 